\title{
Desarrollo de competencias transversales y evaluación de su adquisición en el Grado en Ingeniería Civil
}

Ana María Pérez-Zuriaga ${ }^{a}$, Carlos Llorca García ${ }^{\mathrm{b}}$ y Ana Tsui Moreno Chou

aUniversitat Politècnica de València, anpezu@tra.upv.es, bUniversitat Politècnica de València, carlloga@cam.upv.es 'Universitat Politècnica de València, anmoch@cam.upv.es.

\begin{abstract}
The Vice-Rectorate for Studies, Quality and Acreditation and the Institute of Education Sciences promotes that education is based on competencies, either generic or specific.

The School of Civil Engineering of the Universitat Politècncia de València has chosen the course "Caminos y Aeropuertos" to evaluate the generic competencies "Team working and leadership" and "Effective communication", both oral and written.

The course "Caminos y Aeropuertos" is a core subject in third year of the Deegree in Civil Engineering. The teaching methodology of this technical subject is based on the active participation of students through a diverse set of assignments.

This paper explains the adaptation of the methodology to include the evaluation of generic competencies, having a high number of students (124). It is based on written exercises in teams of 1, 2 and 4 students, as well as on an oral presentation.
\end{abstract}

Keywords: Generic competencies, effective comunication, team-working and leadership, engineering.

\footnotetext{
Resumen

Desde el Vicerrectorado de Estudios, Calidad y Acreditación de la Universitat Politècnica de València, junto con el Instituto de Ciencias de la Educación, se está llevando a cabo un proyecto para dar un giro hacia la formación basada en competencias.

En la Escuela Técnica Superior de Ingenieros de Caminos, Canales y Puertos, se ha elegido la asignatura "Caminos y Aeropuertos" como punto de control para la evaluación de las dimensiones competenciales "Trabajo en Equipo y
} 
Liderazgo" y "Comunicación Efectiva", incluyendo comunicación oral y escrita.

“Caminos y Aeropuertos” es una asignatura de carácter troncal impartida en tercer curso del Grado en Ingeniería Civil, con una base claramente técnica. Desde su concepción, su docencia se ha diseñado con base en metodologías activas. No obstante, ha sido necesario modificar ligeramente su metodología docente para incluir la evaluación de las competencias mencionadas.

En este trabajo se muestra cómo se ha adaptado la metodología docente y cómo se ha procedido a la evaluación de estas competencias dentro de una asignatura eminentemente técnica y con un alto número de alumnos matriculados (124). La metodología se basa principalmente en la realización de trabajos escritos de diferente entidad en grupos de 1, 2 y 4 personas y una exposición oral.

Palabras clave: Competencias transversales, comunicación efectiva, trabajo en equipo y liderazgo, ingeniería.

\section{Introducción}

La Universitat Politèncica de València, al igual que otros muchos centros, fomenta la formación basada en comptencias. Las competencias pueden ser específicas, si se refieren a los distintos elementos o ámbitos particulares de determinadas titulaciones o asignaturas, y transversales, aplicables a cualquier titulación o campo del conocimiento. El documento “Marco UPV de definición y evaluación de adquisición de competencias” (UPV, 2014) define 13 dimensiones competenciales, que deben adquirise en cualquier titulación.

En el Grado en Ingeniería Civil, la Escuela Técnica de Ingenieros de Caminos, Canales y Puertos ha escogido la asignatura "Caminos y Aeropuertos", de tercer curso, como punto de control para la evaluación del grado de adquisición de dos de las dimensiones competenciales establecidas en dicho documento: "Trabajo en equipo y liderazgo" (DC6) y "Comunicación efectiva” (DC8). La primera de ellas se define por la capacidad de trabajar y liderar equipos de forma efectiva para la consecución de objetivos comunes, mientras que la segunda por la capacidad de comunicarse de forma oral y escrita, empleando los recursos necesarios y dirigendose de forma apropiada al receptor.

Otros docentes (Ardid et al, 2014) ya destacaron la oportunidad del trabajo en equipo para adquirir dimensiones competenciales, destacando la comunicación efectiva, y desarrollando una metodología adecuada para su integración en materias eminentemente científicas, como

(c)) EY-NC-ND 2015, Universitat Politècnica de València

Congreso IN-RED (2015) 
el caso de Física. Por su parte, Bañón et al. (2014) plantearon como desagregar la evaluación de una asignatura, ponderando el efecto de cada una de las competencias. Sin embargo, para evaluar las competencias genéricas, de la forma más objetiva posible, la mayoría de autores ha recurrido al uso de rúbricas (Aragón, 2014; De la Poza et al, 2014).

La guía docente de la asignatura "Caminos y Aeropuertos” (UPV, 2014) no especifica la forma en que las competencias genéricas o transversales deben ser incluídas en la metodología docente, tanto en su desarrollo como en su evaluación, ya que la elección de esta asignatura como punto de control de la evaluación de competencias fue posterior a la redacción y publicación de su guía docente. Por lo tanto, en este trabajo se presenta cómo se ha modificado dicha metodología con el objeto de potenciarlas y evaluarlas.

\section{Objetivos}

El principal objetivo de este trabajo es integrar la evaluación de las dimensiones competenciales "Trabajo en Equipo y Liderazgo" (DC.6) y "Comunicación Efectiva" (DC.8), incluyendo comunicación oral y escrita, en la metodología docente de una asignatura eminentemente técnica, como es la asignatura "Caminos y Aeropuertos" del Grado en Ingeniería Civil.

Para ello, se han establecido una serie de objetivos específicos:

- Inclusión en la metodología docente de resolución de problemas en grupos de 2 y 4 estudiantes, complementarios a otros trabajos individuales.

- Inclusión en la metodología docente de exposiciones orales.

- Evaluación de la competencia DC.6 "Trabajo en Equipo y Liderazgo" mediante procesos de coevaluación y autoevaluación.

- Evaluación de la competencia DC.8 "Comunicación efectiva escrita" tanto en los trabajos individuales como en grupos de 2 y 4 personas.

- Evaluación de la competencia DC.8 "Comunicación efectiva oral" en presentaciones orales.

- Comparación de todos los criterios de evaluación anteriores.

\section{Desarrollo}

La metodología docente de la asignatura "Caminos y Aeropuertos" del Grado en Ingeniería Civil se ha adaptado para servir de punto de control en la evaluación del desarrollo de las dimensiones competenciales DC. 6 "Trabajo en Equipo y Liderazgo" y DC.8 "Comunicación Efectiva".

El desarrollo lectivo de la asignatura y la evaluación de la misma se estructura en diferentes actividades prácticas realizadas de forma conjunta mediante grupos de alumnos, pruebas 
objetivas individuales mediante el uso de la herramienta PoliformaT y una prueba escrita final realizada de forma individual.

La evaluación de las competencias transversales se ha centrado en las actividades prácticas en grupo y en la prueba escrita individual.

\subsection{Actividades desarrolladas para la adquisición de las competencias}

La metodología docente desarrollada se basa en 5 ejercicios de clase, 3 trabajos tipo A y 1 trabajo tipo B.

\section{Ejercicios de clase}

Los ejercicios de clase se desarrollan después de la clase magistral correspondiente, cumpliendo así la finalidad de reforzar el contenido impartido en ellas. Cada ejercicio contiene varias cuestiones prácticas sencillas, aumentando su complejidad a lo largo de la sesión.

Para su realización, los alumnos se distribuyen en grupos de 4 personas, definidos previamente de forma aleatoria por el profesor. Una vez en grupo, resuelven los ejercicios planteados y entregan la solución al finalizar la clase.

Durante los ejercicios se cuenta con tres docentes en el aula para resolver las dudas del ejercicio o de la teoría impartida.

Con esta metodología se han desarrollado 4 de los 5 ejercicios, mientras que el quinto se ha llevado a cabo de forma individual por las características del mismo.

\section{Trabajos tipo A}

Los trabajos tipo A son actividades no presenciales que requieren un máximo de 3 horas de trabajo del alumno para su preparación.

En el caso de la metodología planteada, los trabajos se comienzan en el aula y se finalizan fuera del horario lectivo, todo ello en grupos de 2 personas, entregándolos una semana después.

Cada trabajo tipo A representa un problema de mayor complejidad que los desarrollados en los ejercicios, pues implica un trabajo de análisis y el diseño de soluciones sencillas.

\section{Trabajo tipo B}

Los trabajos tipo B son actividades no presenciales que requieren entre 3 y 6 horas de trabajo del alumno para su preparación.

En el caso de este trabajo, se desarrolla en su totalidad fuera del aula en grupos de 2 personas y se entrega varios días después de la prueba escrita. El trabajo tipo B representa un problema 
todavía más complejo que los trabajos tipo A, e implica el análisis, diagnóstico y el planteamiento de soluciones originales, así como la redacción de un informe.

\section{Exposición oral}

Tras la corrección de los ejercicios de clase y de los trabajos tipo A, los profesores, teniendo en cuenta los resultados de los mismos, designan los grupos de 4 personas que tienen que realizar una presentación oral sobre cada uno de ellos.

Estas presentaciones se han llevado a cabo en la sesión anterior a la prueba escrita. Se optó por hacerlo durante la sesión inmediatamente anterior a la prueba escrita ya que con las presentaciones se realiza una revisión de todos los ejercicios y trabajos tipo A desarrollados, repasando así toda la asignatura.

\section{Prueba escrita}

La prueba escrita, realizada de forma individual, engloba una parte teórica, con una cuestión similar a los ejercicios realizados y con una pregunta puramente teórica, y una parte práctica con un problema similar a los realizados en los trabajos tipo A.

\subsection{Evaluación de las competencias}

Cada una de las anteriores actividades se evalúa, en primer lugear, de la forma habitual, obteniendo una nota de 1 a 10 en cada una de ellas. Paralelamente, pero de forma independiente, se realiza la evaluación de las competencias transversales.

La evaluación de la competencia DC.6 "Trabajo en Equipo y Liderazgo" se realiza al finalizar los ejercicios de clase por parte de los alumnos mediante evaluación y coevaluación, siguiendo la rúbrica proporcionada por los profesores. El alumno debe asignar a cada uno de los ítems, a cada uno de los miembros del grupo, la calificación de 1 a 5, no pudiendo repetirse valores dentro del grupo. De esta manera, se evita una posible laminación de la coevaluación. La coevaluación y autoevaluación tiene carácter voluntario y no tiene repercusión en la nota de los alumnos evaluados.

Por otra parte, la evaluación de la competencia DC.8 "Comunicación efectiva" se divide en comunicación efectiva oral y escrita. Ambas evaluaciones son realizadas únicamente por los profesores, por lo que abarcan a la totalidad de los alumnos. La evaluación de la comunicación efectiva escrita se realiza tanto sobre los ejercicios realizados por 4 personas, como sobre los trabajos realizados en parejas y sobre la prueba escrita realizada de forma individual. De esta forma puede observarse las diferencias entre el trabajo individual y el colectivo. En caso de los trabajos colectivos, la nota es única para todo el equipo. 
La evaluación de la comunicación oral se hace sobre la exposición oral que los alumnos hacen al final de la asignatura, antes de la prueba escrita. La evaluación es individual para cada alumno y fue realizada durante la exposición.

En la tabla 1 se recoge un resume de las rúbricas para la evaluación de las dimensiones competencionales de las que es punto de control la asignatura "Caminos y Aeropuertos". Cada uno de los ítems de la rúbrica que se mencionan en esta tabla tiene una gradación de 5 niveles, perfectamente definidos. Así, se facilita una evaluación de las competencias lo más objetiva posible.

Tabla 1. Resumen de las rúbricas para la evaluación de competencias genéricas

\begin{tabular}{|c|c|c|}
\hline $\begin{array}{c}\text { Dimensión a } \\
\text { evauar }\end{array}$ & Evaluador & Ítems de la rúbrica \\
\hline $\begin{array}{c}\text { DC.6: Trabajo } \\
\text { en equipo y } \\
\text { liderazgo }\end{array}$ & Alumno & $\begin{array}{l}\text { - Realiza tareas en plazo. } \\
\text { - Participa de forma activa. } \\
\text { - Contribuye a la cohesión del equipo. } \\
\text { - Contribuye a definir los procesos de trabajo. } \\
\text { - } \text { Dirige las reuniones con eficacia. } \\
\text { - Propone metas ambiciosas pero concisas. } \\
\text { - Facilita la gestión positiva de conflictos. }\end{array}$ \\
\hline $\begin{array}{c}\text { DC.8: } \\
\text { Comunicación } \\
\text { efectiva escrita }\end{array}$ & Profesor & $\begin{array}{l}\text { - Expresión, gramática y puntuación. } \\
\text { - Ortografía. } \\
\text { - Terminología. } \\
\text { - Orden y organización. } \\
\text { - Responde a lo que se pregunta. }\end{array}$ \\
\hline $\begin{array}{c}\text { DC.8: } \\
\text { Comunicación } \\
\text { efectiva escrita }\end{array}$ & Profesor & $\begin{array}{l}\text { - } \quad \text { Conocimiento del tema. } \\
\text { - } \text { Lenguaje apropiado. } \\
\text { - } \quad \text { Capacidad de comunicación y convicción. } \\
\text { - Modulación de la voz. } \\
\text { - Lenguaje no verbal y nervisosismo. }\end{array}$ \\
\hline
\end{tabular}

Con todo ello, quedan evaluadas las dimensiones competenciales de las que la asignatura "Caminos y Aeropuertos" es punto de control en el Grado de Ingeniería Civil. 
La asignatura se imparte en 3 grupos de 65, 28 y 31 alumnos. Durante los ejercicios y explicación de las prácticas se cuenta con un profesor, un profesor universitario en formación (FPU) y un investigador en formación (FPI).

\section{Resultados}

Los resultados obtenidos se estructuran tal como se describe a continuación. En primer lugar, se analiza la evaluación de cada una de las competencias transversales estudiadas, de forma independiente. En concreto, se describen la muestras de evaluaciones obtenidas para el conjunto de alumnos matriculados. Posteriormente, se analizan las correlaciones existentes entre la evalaución de competencias transversales y las notas de las actividades realizadas por los alumnos.

\subsection{Trabajo en equipo y liderazgo}

Tal como se ha comentado, la evaluación de la competencia genérica DC.6 “Trabajo en equipo y liderazgo” se realizó por medio de la autoevaluación de cada uno de los miembros del grupo a sus compañeros. Esta coevaluación se centró en los ejercicios de clase 1.1, 1.2 y 1.3, dado que son las únicas actividades realizadas en grupos de 4. Posteriormente, se realizó otro ejercicio en grupos de 4 pero en él los alumnos no llevaron a cabo esta evaluación de competencias.

Esta coevaluación no forma parte de la nota de los alumnos, y por tanto, tuvo carácter voluntario. No obstante, se insistió en la gran importancia que tenía la correcta cumplimentación de las hojas de coevaluación. Por todo ello, en primer lugar se analiza la participación de los alumnos en esta tarea (tabla 2 y tabla 3).

Tabla 2. Participación en la coevaluación-autoevaluación de la competencia DC6

\begin{tabular}{cccccc}
\hline \multirow{2}{*}{ Grupo } & \multirow{2}{*}{ Alumnos } & \multicolumn{4}{c}{ Participación } \\
\cline { 3 - 6 } & & E1.1 & E1.2 & E1.3 & Total \\
\hline F & 67 & 51 & 48 & 24 & 123 \\
G & 28 & 13 & 13 & 6 & 32 \\
H & 29 & 15 & 13 & 8 & 36 \\
Total & 125 & 79 & 74 & 38 & 191 \\
\hline
\end{tabular}

(c)) EY-NC-ND 2015, Universitat Politècnica de València

Congreso In-Red (2015) 
Desarrollo de competencias transversales y evaluación de su adquisición en el Grado en Ingeniería Civil

Tabla 3. Representatividad de la coevaluación-autoevaluación de la competencia DC6

\begin{tabular}{ccc}
\hline $\begin{array}{c}\text { Número de veces } \\
\text { evaluado por sus } \\
\text { compañeros }\end{array}$ & $\begin{array}{c}\text { Número de alumnos que reciben } \\
\text { dicha autoevaluación }\end{array}$ & $\begin{array}{c}\text { Porcentaje sobre el } \\
\text { total }\end{array}$ \\
\hline 0 & 8 & $6 \%$ \\
1 & 18 & $14 \%$ \\
2 & 35 & $28 \%$ \\
3 & 41 & $33 \%$ \\
4 & 23 & $18 \%$ \\
\hline
\end{tabular}

La Tabla 2 recoge el número de alumnos que han participado en esta coevaluación. El porcentaje global de evaluaciones completadas es del 50\% (191 evaluaciones realizadas sobre un total de 125 x 3 posibles evaluaciones). Dado el carácter voluntario de esta actividad evaluadora, se percibe además que la participación decrece desde el ejercicio 1.1 (63\%) hasta el $1.3(30 \%)$.

La Tabla 3, por su parte, se centra en la representatividad de los resultados. Entendiendo que la evaluación de la competencia es más representativa cuando más miembros del equipo la han realizado, se observa que el 51\% de los alumnos recibieron, al menos, 3 evaluaciones distintas. Un 79\% de los alumnos recibieron, al menos, dos evaluaciones.

La rúbrica planteada tenía un total de siete ítems, cuyas notas medias se situaban entre 3,6 y 3,7. De esto se deduce que la evaluación que realizan los alumnos sobre sus compañeros no es muy diferente en cada uno de los ítems, lo que sugiere no tratarlos por separado.

El rango, o diferencia entre la media más alta y la más baja de las notas medias recibidas por cada alumno se ha asimilado como medida de la dispersión, y por tanto de la consistencia entre las distintas evaluaciones recibidas por cada alumno.

Tabla 4. Consistencia en la coevaluación de la competencia DC.6

\begin{tabular}{ccc}
\hline Intervalo de rango & Número de casos & Porcentaje \\
\hline 0 a 0.5 & 44 & $34 \%$ \\
0.5 a 1 & 32 & $25 \%$ \\
1 a 1.5 & 17 & $13 \%$ \\
1.5 a 2 & 3 & $2 \%$ \\
2 a 2.5 & 1 & $1 \%$ \\
2.5 a 3 & 2 & $2 \%$ \\
3 a 3.5 & 0 & $0 \%$ \\
\hline
\end{tabular}


Tal como se deduce de la Tabla 4, en más de la mitad de los casos, la diferencia entre la media más alta y la más baja es inferior a 1 punto. Este hecho da validez a la evaluación de la competencia DC.6, al mostrar un elevado grado de acuerdo entre los distintos coevaluadores. Una vez estudiado esto, la Fig. 1 muestra la distribución de las notas medias, agregadas en intervalos, de la competencia transversal DC.6. Tal como puede apreciarse, las calificaciones obtenidas en la co-evaluación se centran principalmente en los valores altos: 3,4 y 5 , y son pocos los casos en los que se asignan las calificaciones inferiores.

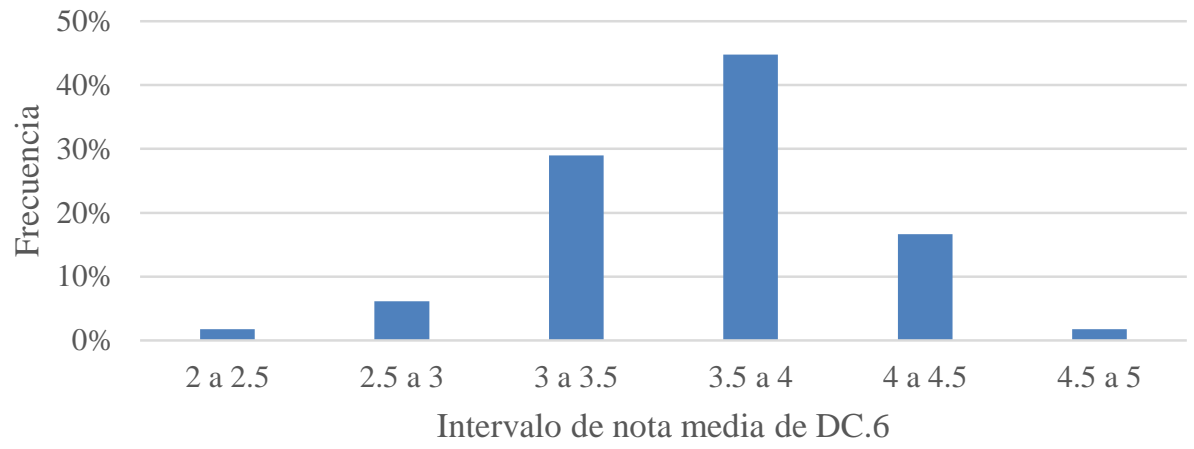

Fig. 1. Distribución de notas medias en la competencia DC.6

\subsection{Comunicación efectiva oral}

La evaluación de la competencia DC.8 se ha desglosado en comunicación oral y comunicación escrita. En cuanto a la competencia de comunicación efectiva oral, se ha realizado una valoración de la calidad de una presentación oral acerca de una de las actividades prácticas de la asignatura. Esta valoración tenía carácter voluntario y es de tipo individual.

En la Tabla 5 se presenta el tamaño de la muestra de evaluaciones de la competencia de comunicación efectiva oral, que llega al 75\% de los alumnos.

Tabla 5. Participación en la evaluación de la competencia DC.8 (comunicación oral).

\begin{tabular}{ccc}
\hline Grupo & Alumnos & $\begin{array}{c}\text { Alumnos que han realizado } \\
\text { presentación oral }\end{array}$ \\
\hline F & 67 & 59 \\
G & 28 & 19 \\
H & 29 & 15 \\
Total & 125 & 93 \\
\hline
\end{tabular}

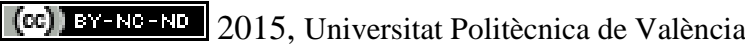

Congreso In-Red (2015) 


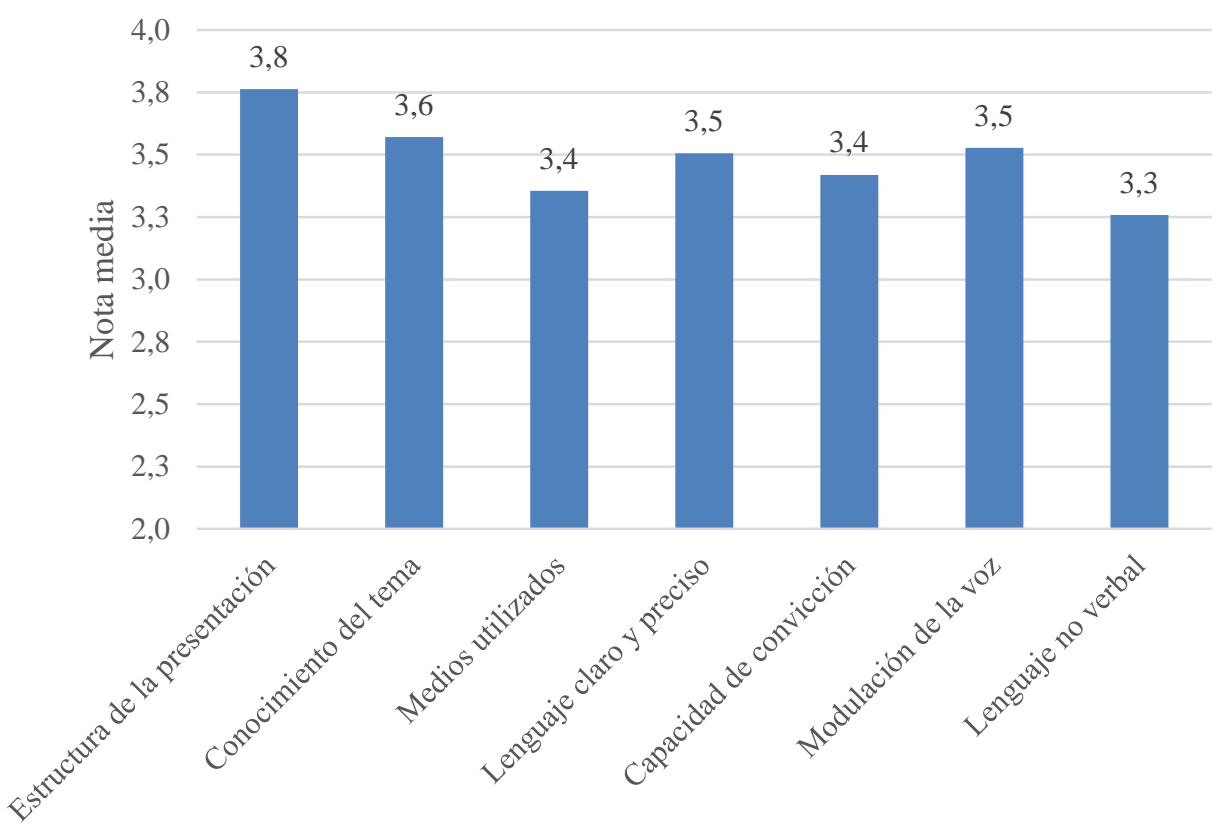

Fig. 2. Resumen de notas medias de la competencia DC.8 (comunicación efectiva oral)

Las diferencias en los valores medios de los ítems de evaluación de la comunicación efectiva oral muestran una dispersión ligeramente mayor. En concreto, las peores valoraciones se obtuvieron en los ítems "medios utilizados”, “capacidad de convicción” y "lenguaje no verbal”. La valoración más alta se alcanzó en el ítem “estructura de la presentación”.

\subsection{Comunicación efectiva escrita}

Por su parte, la comunicación efectiva escrita se evaluó para un mayor número de actividades prácticas, y en la prueba escrita final de la asignatura. En este apartado, se desglosa la evaluación en actividades en equipos de 4 y 2 miembros y en las individuales.

Tabla 6. Muestra de la evaluación de la competencia DC.8 (comunicación escrita).

\begin{tabular}{cccc}
\hline Grupo & Grupos (Ejercicios & Parejas (Trabajos & Prueba escrita \\
\hline F & 1.1 - 1.3) & A 2.1 - 2.3) & \\
G & 7 & 33 & 99 \\
H & 7 & 11 & \\
Total & 34 & 13 & \\
\hline
\end{tabular}


En la Tabla 6 se recoge el tamaño de la muestra correspondiente a cada tipo de actividad práctica o de evaluación. Cada uno de los totales es igual al doble del anterior, puesto que se pasa de grupos de 4 alumnos, a parejas y a exámenes individuales.

La Fig. 3 resume las notas medias de cada uno de los ítems de la rubrica. No se aprecia, en general, una gran variabilidad entre los valores medios de cada ítem, si bien la valoración media en cada tipo de actividad sí que es diferente. En este sentido, y también en la Fig. 4, se observa que la evaluación de la competencia escrita es mejor en el caso de los ejercicios de grupo, respecto de las prácticas por parejas y respecto del examen. Esta diferencia puede deberse al hecho de que los ejercicios en grupo son actividades de aplicación directa de conceptos, y en las que la expresión escrita puede no ser una competencia tan significativa como en otras actividades con mayor desarrollo por parte del alumno. Además, se presentaban en grupo y el alumno con mejor expresión podría ser el encargado de su redacción.

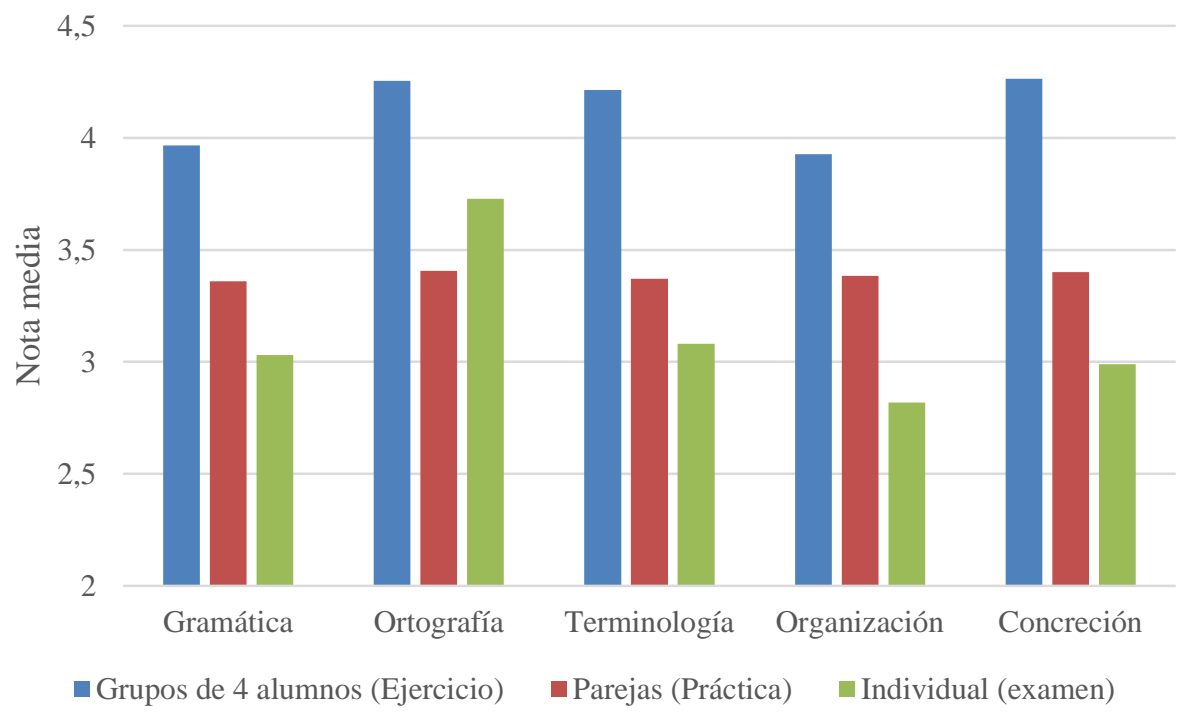

Fig. 3. Resumen de las notas medias de la competencia DC.8 (comunicación efectiva escrita) 
Desarrollo de competencias transversales y evaluación de su adquisición en el Grado en Ingeniería Civil

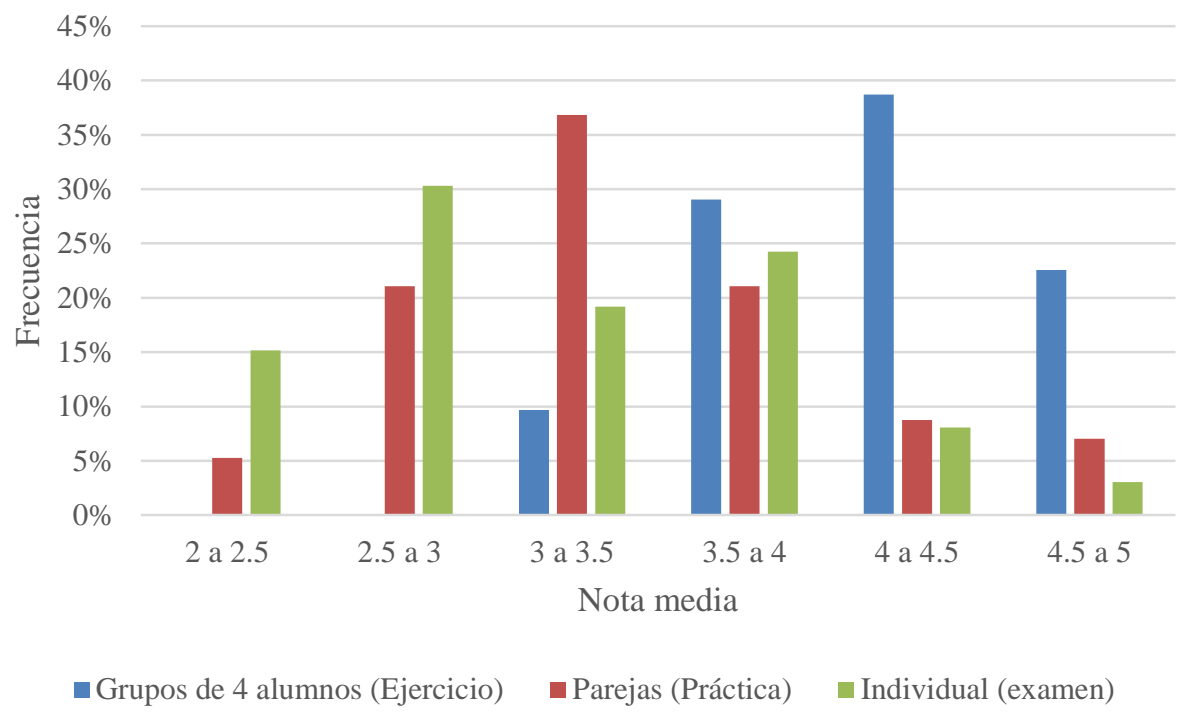

Fig. 4. Distribución de evaluaciones medias de la competencia DC.8 (comunicación efectiva escrita)

Igualmente, en la Fig. 5 se presentan las correlaciones entre las notas medias de cada tipo de actividad, y las correspondientes medias en la evaluación de la competencia DC.8, comunicación efectiva escrita, de cada uno de los grupos, parejas o alumnos. Se aprecia, en el caso de los ejercicios en grupo, que ambas evaluaciones se sitúan en el tercio superior de la escala y que no se relacionan entre sí. Esta relación sí que es muy significativa en las prácticas por parejas (R-cuadrado de 61\%) y en el examen individual (R-cuadrado de 48\%) en las que se detecta un rango más amplio de calificaciones. 

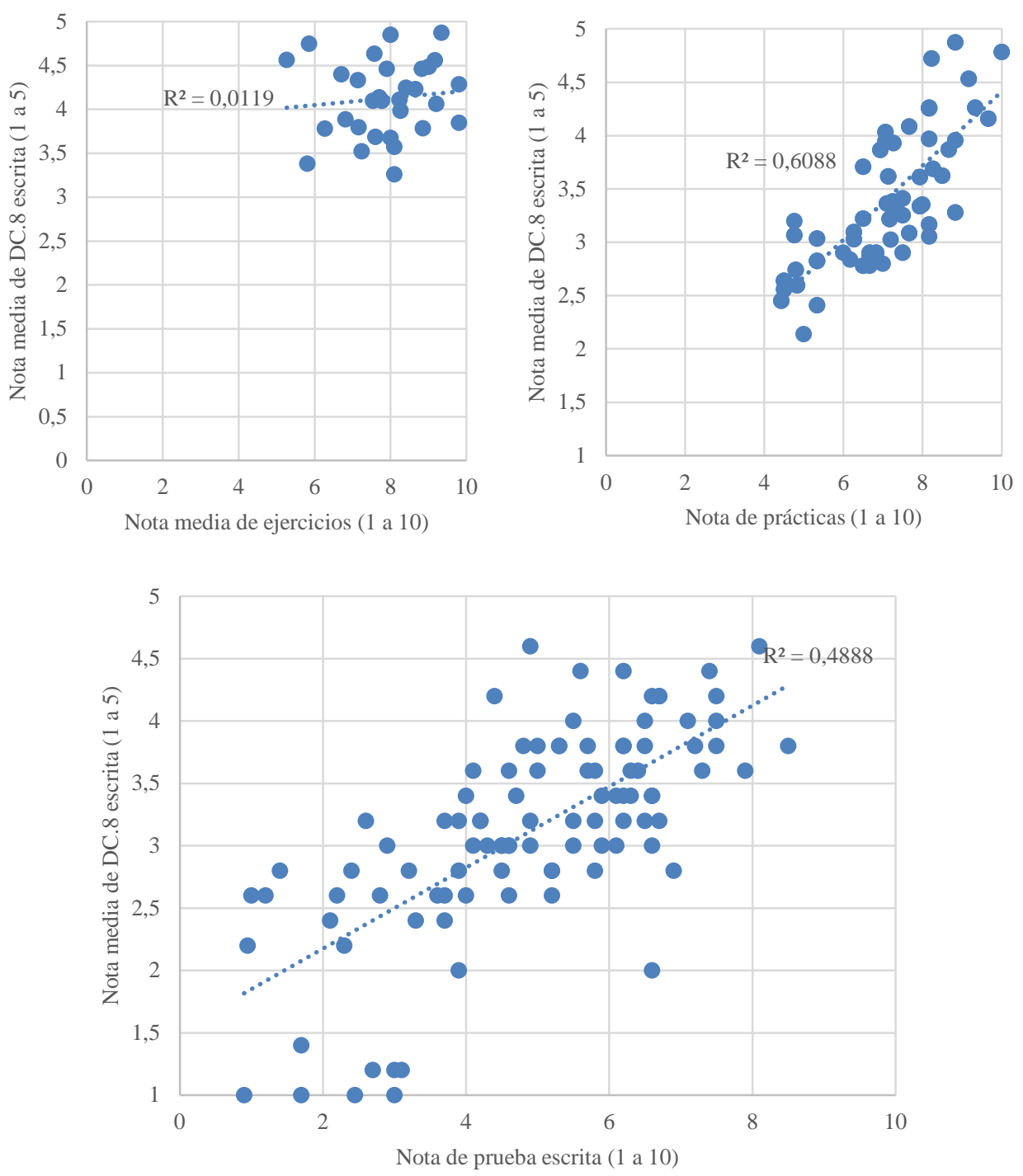

Fig. 5. Relación entre la nota media de la actividad y la correspondiente nota media de la competencia DC.8 escrita

\section{Conclusiones}

En la asignatura "Caminos y Aeropuertos” del tercer curso del Grado en Ingeniería Civil se han evaluado las competencias transversales "Trabajo en equipo y liderazgo" (DC6) y “Comunicación efectiva” (DC8). Se pueden extraer las siguientes conclusiones:

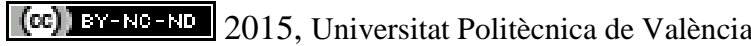

Congreso In-Red (2015) 
- $\quad$ La participación media de los alumnos en la coevaluación fue del 50 \%. Además, la participación decreció conforme avanzaba la asignatura, hasta el 30 \%. El carácter voluntario de esta actividad evaluadora no implicó en exceso a los alumnos pese a insistir en su importancia.

- Las calificaciones de los alumnos por coevaluación de la competencia “Trabajo en equipo y liderazgo" son generalmente elevadas y con poca dispersión entre los distintos ítems. Por un lado, muestra elevado grado de acuerdo entre los distintos coevaluadores y, por otro lado, poca diferencia entre los ítems de la rúbrica. Puede ser que los alumnos califiquen globalmente a sus compañeros y le asignen la misma nota a todos los campos.

- $\quad$ La participación de los alumnos en las presentaciones orales fue elevada, del 75 \%. Todo ello a pesar de que la presentación oral era voluntaria. Esto indica que los alumnos entendieron la importancia de participar en estas presentaciones para revisar el contenido de toda la asignatura antes de la prueba escrita.

- A nivel general, los alumnos estructuraron de manera satisfactoria las presentaciones orales, si bien la capacidad de convicción y el lenguaje no verbal obtuvieron las peores calificaciones. Podrían ser necesarias más sesiones donde los alumnos expongan su trabajo o incluir más directrices para la preparación de la presentación.

- Dentro de la expresión escrita, los ítems de organización y terminología suelen recibir la menor calificación en todas las actividades (ejercicios de grupo, trabajos tipo A y B y prueba escrita).

- La expresión escrita de los alumnos se correlaciona con la calificación de la actividad en cierta medida en la prueba escrita (R2=49\%) y en los trabajos por parejas (R2=60 \%). En los ejercicios en grupo, debido al mayor número de participantes y a la mayor concreción de las preguntas, la expresión escrita no es tan significativa y guarda menor relación con la nota.

A partir de la experiencia en la asignatura "Caminos y Aeropuertos”, se recomienda para la evaluación de competencias transversales:

- Simplificar la rúbrica de la competencia “trabajo en equipo y liderazgo". Los alumnos tienden a dar la puntuación a sus compañeros en función de su percepción global.

- Evaluar las competencias de comunicación efectiva oral con más de un docente o mediante video. Es complejo evaluar tanto el contenido de la presentación como todos los ítems de todos los alumnos de forma simultánea. 
- Incluir más de una presentación oral por alumno, si el tamaño de la clase lo permite. En el caso particular de la asignatura objeto de estudio, debido a la gran cantidad de alumnos (124 alumnos), podría considerarse como una actividad complementaria fuera del horario de la asignatura.

- Incidir en la importancia de una adecuada organización del texto y la adecuación de la terminología. Estos dos aspectos son los que menor puntuación recibieron, de forma consistente, en las actividades.

- Involucrar a los alumnos en la evaluación de las competencias transversales, enfatizando la importancia de la coevaluación. Existe una considerable pérdida de alumnos evaluando si no conlleva un incremento en la nota global de la asignatura.

\section{Referencias}

ARAGON, P. (2014). "Diseño y evaluación de una actividad de aprendizaje basado en proyectos en el contexto de la Higiene analítica” en Jornadas In-Red. Valencia.

ARDID M., CUENCA, V.P., MESEGUER, J.M., MOLINA, J. RIERA, J., SALINAS, I y VIDAURRE, A. (2014). “ Trabajo en equipo: una oportunidad para adquirir dimensiones competenciales” en Jornadas In-Red. Valencia.

BAÑON, A.J., CLEMENTE, L, CORTÉS, N, LAJARA, J.M., MONTALVÁ, J.M., ROVIRA, A., TRINIDAD, J.J. y VILLANUEVA, J.F. (2014). “Comunicación efectiva: Un enfoque para distintas asignaturas de la UPV” en Jornadas In-Red. Valencia.

DE LA POZA, E., DOMENECH, J., GUADALAJARA, N.., MÁS, F, MARTÍNEZ, V., BARRACHINA, I., MARTÍ, M.L. y VIVAS, D. (2014). "La co-evaluación como sistema de evaluación del trabajo colaborativo” en Jornadas In-Red. Valencia.

UNIVERSITAT POLITÈCNICA DE VALÈNCIA (2014). Guía docente de la asignatura "Caminos y Aeropuertos".

UNIVERSITAT POLITÈCNICA DE VALÈNCIA (2014). Marco UPV de definición y evaluación de adquisición de competencias. 